Optical Society of America. Yongxin Wang, Ming Han, and Anbo Wang, "Analysis of a high-speed fiber-optic spectrometer for fiber-optic sensor signal processing," Appl. Opt. 46, 8149-8158 (2007). doi: 10.1364/ ao. 46.008149

\title{
Analysis of a high-speed fiber-optic spectrometer for fiber-optic sensor signal processing
}

\author{
Yongxin Wang, ${ }^{*}$ Ming Han, and Anbo Wang \\ Bradley Department of Electrical and Computer Engineering, Center for Photonics Technology, \\ Virginia Polytechnic Institute and State University, Blacksburg, Virginia 24061-0111, USA \\ *Corresponding author: ywang1@vt.edu
}

Received 1 June 2007; revised 10 August 2007; accepted 11 September 2007; posted 5 October 2007 (Doc. ID 83678); published 19 November 2007

\begin{abstract}
A novel high-speed fiber-optic spectrometer has been demonstrated in our previous work. The high-speed spectrum measurement is enabled by translating the spectral-domain signal into a time-domain signal through a dispersion element. We present a mathematical model that accurately describes the relationship between the optical spectrum to be measured and the dispersed time-domain signal. Based on the model, the effects of the key parameters on the performance of the spectrometer are investigated in detail using numerical simulation. The analysis is useful for the design and application of such spectrometers. (C) 2007 Optical Society of America
\end{abstract}

OCIS codes: $\quad 300.6190,300.6530,120.6200,060.2370,060.5530$.

\section{Introduction}

An optical spectrometer is an instrument that measures the spectral density of light over a certain part of the electromagnetic spectrum. Optical spectrometers have been widely used for the signal demodulation of interferometric and grating-based fiber-optic sensors. In these systems, the signals are the spectra of a broadband light from the sensor that are recorded by a spectrometer [1-3]. For interferometric sensors, the absolute value of the optical path difference between the two arms or the cavity length of the interferometer can be determined by the spectral positions of the peaks and valleys in the sensor interference fringes [1] or through a certain fitting algorithm [4] so that the corresponding measurands are demodulated. In contrast to fringe counting or quadrature phase detection, these methods offer absolute measurement yet with high resolution and accuracy. For fiber grating sensors, the reflection wavelength usually gives a direct indication of the measurand. Generally, owing to their small sizes, the sensor elements of many fiber-optic sensors have an intrinsically high-speed response, but their signal demodulation

0003-6935/07/338149-10\$15.00/0

(C) 2007 Optical Society of America often limits the system speed due to the low-speed spectrum measurement.

There are several spectrum measurement techniques. The most popular one is using a dispersive device, e.g., a prism or a diffraction grating, to spatially spread the light by its wavelength. Then at the exit of the spectrometer, a single detector or a detector array is utilized to record the dispersed light to realize spectrum measurement. Some spectrometers also tune a narrow bandpass filter to scan the spectrum, whereas Fourier transform spectrometers acquire the interferogram generated by an interferometer and perform the Fourier transform of the interferogram so that the spectral density of the incoming light can be recovered.

We have experimentally demonstrated a highspeed fiber-optic spectrometer (HSFOS) recently for fiber-optic sensors [5]. In this paper, we develop a mathematical model for detailed analysis of such a spectrometer. The analysis is useful for the design and application of such spectrometer. The remainder of the paper is organized as follows. In Section 2, a mathematical model that describes the relationship between the spectrum and the time-domain signal is presented. Based on the model, performance of the spectrometer, including the accuracy, the spectral resolution, and the measurement speed are analyzed, 
respectively, in Section 3. Finally some conclusions are given in Section 4.

\section{Mathematical Model}

The operational principle of the HSFOS is illustrated in Fig. 1. The incoming light signal transmitted to the HSFOS is first modulated by a series of narrow pulses with fixed pulse width and repetition rate from the pulse generator through the modulator to form the time-domain samples of the light signal, each of which will finally form one frame of the spectrum. These samples go through a span of dispersive fiber so that the time-domain dispersion is achieved. Each dispersed pulse signal has a profile similar to the spectrum of the incoming light. Then the optical-toelectric $(\mathrm{O} / \mathrm{E})$ converter converts the light signal to the electric signal and the high-speed data acquisition device (DAQ) samples and digitizes the signal and then sends the data to a computer for spectrum construction.

Ignoring the fiber nonlinearity, the whole process presented above can be mathematically modeled as follows. The field $E(0, t)$ and the intensity $I(0, t)$ of the incoming light are described as

$$
\begin{aligned}
E(0, t) & =A(t) \exp \left(j \omega_{0} t\right), \\
I(0, t) & =|E(0, t)|^{2}=E(0, t) E^{*}(0, t),
\end{aligned}
$$

where $A(t)$ is the complex amplitude and “*” denotes complex conjugate. The complex amplitude, $A(t)$, is assumed to be a stationary random process. Then the spectral density function $P(0, \omega)$ is expressed as

$$
\begin{aligned}
P(0, \omega)= & \frac{1}{(2 \pi)^{2}} \int_{-\infty}^{+\infty} \int_{-\infty}^{+\infty} E(0, t) E^{*}\left(0, t^{\prime}\right) \\
& \times \exp \left[-j \omega\left(t-t^{\prime}\right)\right] \mathrm{d} t \mathrm{~d} t^{\prime} .
\end{aligned}
$$

For intensity modulators, define $M_{0}(t)$ as the intensity modulation signal, and $m_{0}(t)$ is the square root of the modulation signal; for amplitude modulators, define $m_{0}(t)$ as the modulation signal. The spectrum of the modulation signal is

$$
F_{0}(\omega)=\frac{1}{2 \pi} \int_{-\infty}^{+\infty} m_{0}(t) \exp (-j \omega t) \mathrm{d} t
$$

Following Marcuse's previous works [6-8], the profile of the light signal $s(t)$ after propagation through

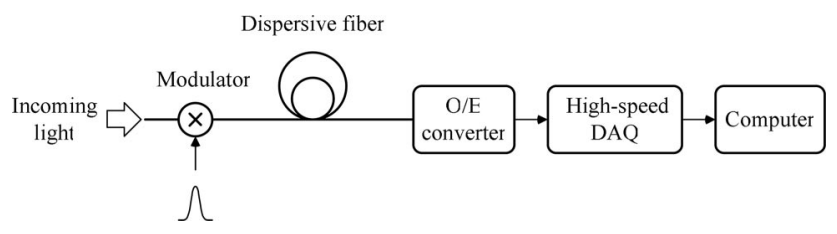

Fig. 1. Operational principle of the HSFOS. the dispersive fiber is given by

$$
\begin{aligned}
s(t)= & \int_{-\infty}^{+\infty} P\left(0, \omega^{\prime}\right) \mid \int_{-\infty}^{+\infty} F_{0}\left(\omega-\omega^{\prime}\right) \exp \left\{j \left[\left(\omega-\omega^{\prime}\right) t\right.\right. \\
& \left.\left.-\left(\beta-\beta^{\prime}\right) L\right]\right\}\left.\mathrm{d} \omega\right|^{2} \mathrm{~d} \omega^{\prime} .
\end{aligned}
$$

The propagation constant $\beta(\omega)$ in the above equation could be expanded in a Taylor series about the circular frequency $\omega^{\prime}$,

$$
\beta(\omega)=\sum_{m=0}^{\infty} \frac{\beta_{m}\left(\omega^{\prime}\right)}{m !}\left(\omega-\omega^{\prime}\right)^{m},
$$

where

$$
\beta_{m}=\frac{\mathrm{d}^{m} \beta}{\mathrm{d} \omega^{m}} \quad(m=0,1,2, \ldots) .
$$

In previous work, the propagation constant is usually expanded around the center circular frequency $\omega_{0}$. The advantages of using $\omega^{\prime}$ instead of $\omega_{0}$ are that, as shown later in this section, a clearer picture of how a chromatic light pulse is disperse in the dispersive fiber could be formed and a more concise expression could be acquired that makes both analytic and numerical analysis easier. From Eq. (6) we have

$$
\beta(\omega)-\beta\left(\omega^{\prime}\right)=\left(\omega-\omega^{\prime}\right) \beta_{1}\left(\omega^{\prime}\right)+\gamma\left(\omega, \omega^{\prime}\right),
$$

where

$$
\gamma\left(\omega, \omega^{\prime}\right)=\sum_{m=2}^{\infty} \frac{\beta_{m}\left(\omega^{\prime}\right)}{m !}\left(\omega-\omega^{\prime}\right)^{m} .
$$

In Eq. (8), $\beta_{1}\left(\omega^{\prime}\right)$ is related to the group velocity of the monochromatic light at $\omega^{\prime}$. Substituting Eq. (8) into Eq. (5), we obtain

$$
\begin{aligned}
s(t)= & \int_{-\infty}^{+\infty} P\left(0, \omega^{\prime}\right) \mid \int_{-\infty}^{+\infty} F_{0}\left(\omega-\omega^{\prime}\right) \\
& \times\left.\exp \left\{j\left[\left(\omega-\omega^{\prime}\right)\left(t-\beta_{1}\left(\omega^{\prime}\right) L\right)-\gamma\left(\omega, \omega^{\prime}\right) L\right]\right\} \mathrm{d} \omega\right|^{2} \mathrm{~d} \omega^{\prime} .
\end{aligned}
$$

Let $\omega^{\prime \prime}=\omega-\omega^{\prime}$, then

$$
\gamma\left(\omega, \omega^{\prime}\right)=\Gamma\left(\omega^{\prime \prime}, \omega^{\prime}\right)=\sum_{m=2}^{\infty} \frac{\beta_{m}\left(\omega^{\prime}\right)}{m !} \omega^{\prime \prime m} .
$$


Define

$$
\begin{gathered}
F\left(L, \omega^{\prime \prime}, \omega^{\prime}\right)=F_{0}\left(\omega^{\prime \prime}\right) \exp \left(-j \Gamma\left(\omega^{\prime \prime}, \omega^{\prime}\right) L\right) \\
t_{d}\left(\omega^{\prime}\right)=\beta_{1}\left(\omega^{\prime}\right) L .
\end{gathered}
$$

Equation (10) now becomes

$$
\begin{aligned}
s(t)= & \int_{-\infty}^{+\infty} P\left(0, \omega^{\prime}\right) \mid \int_{-\infty}^{+\infty} F\left(L, \omega^{\prime \prime}, \omega^{\prime}\right) \\
& \times\left.\exp \left\{j \omega^{\prime \prime}\left(t-t_{d}\left(\omega^{\prime}\right)\right)\right\} \mathrm{d} \omega^{\prime \prime}\right|^{2} \mathrm{~d} \omega^{\prime} .
\end{aligned}
$$

It should be noticed that $F\left(L, \omega^{\prime \prime}, \omega^{\prime}\right)$ in Eq. (14) is the spectrum of a monochromatic light modulated by pulse signal $m_{0}(t)$ and then dispersed by the dispersive fiber. The term $t_{d}$ in Eq. (14) is the group delay of the broadened light pulse and it is a function of circular frequency $\omega^{\prime}$, which is the center circular frequency of the light pulse. With the following relations,

$$
\begin{aligned}
\omega^{\prime}\left(t_{d}\right) & =t_{d}^{-1}\left(\omega^{\prime}\right), \\
\mathrm{d} \omega^{\prime} & =\frac{\mathrm{d} t_{d}}{t_{d}\left(\omega^{\prime}\right)}=\frac{\mathrm{d} t_{d}}{\beta_{2}\left(\omega^{\prime}\right) L},
\end{aligned}
$$

where $t_{d}^{-1}\left(\omega^{\prime}\right)$ represents the inverse function of the function $t_{d}\left(\omega^{\prime}\right)$ and the dot over $t_{d}$ in Eq. (16) represents the derivative, and defining

$$
m\left(L, t, t_{d}\right)=\int_{-\infty}^{+\infty} F_{t}\left(L, \omega^{\prime \prime}, t_{d}\right) \exp \left(j \omega^{\prime \prime}\left(t-t_{d}\right)\right) \mathrm{d} \omega^{\prime \prime},
$$

$$
M\left(L, t, t_{d}\right)=\left|m\left(L, t, t_{d}\right)\right|^{2},
$$

where $F_{t}\left(L, \omega^{\prime \prime}, t_{d}\right)=F\left(L, \omega^{\prime \prime}, \omega^{\prime}\right)$, Eq. (14) becomes

$$
s(t)=\int_{-\infty}^{+\infty} \frac{P_{t}\left(0, t_{d}\right)}{\beta_{2 t}\left(t_{d}\right) L} M\left(L, t, t_{d}\right) \mathrm{d} t_{d},
$$

with $\beta_{2 t}\left(t_{d}\right)=\beta_{2}\left(\omega^{\prime}\right), P_{t}\left(0, t_{d}\right)=P\left(0, \omega^{\prime}\right)$. Setting

$$
P_{n}\left(0, t_{d}\right)=\frac{P_{t}\left(0, t_{d}\right)}{\beta_{2 t}\left(t_{d}\right) L}=\frac{P\left(0, \omega^{\prime}\right)}{\beta_{2}\left(\omega^{\prime}\right) L},
$$

Eq. (19) could be rewritten as

$$
s(t)=\int_{-\infty}^{+\infty} P_{n}\left(0, t_{d}\right) M\left(L, t, t_{d}\right) \mathrm{d} t_{d} .
$$

In the above equation, $s(t)$ is presented as an output corresponding to the input $P_{n}\left(0, t_{d}\right)$ from a linear time variant (LTV) system whose impulse response is described by $M\left(L, t, t_{d}\right)$. The input signal $P_{n}\left(0, t_{d}\right)$ is directly related to the light spectrum to be measured through Eq. (20). The function $M\left(L, t, t_{d}\right)$, which represents the shape of the monochromatic light pulse broadened by the dispersive fiber and is a function of $t_{d}$, shows that at different time points $t_{d}$ or equivalently at different circular frequencies or wavelengths, the broadening of the monochromatic light pulse could be different. Further the $\Gamma\left(\omega^{\prime \prime}, \omega^{\prime}\right)$ term defined in Eq. (11) determines the broadening of the pulses according to the frequency $\omega^{\prime}$. The group delay for each pulse $t_{d}$ defined in Eq. (13) is also related to the circular frequency of the corresponding monochromatic light $\omega^{\prime}$. Thus the output of the spectrometer $s(t)$ can be treated as the superposition of all the frequency-dependent broadened and delayed monochromatic light pulses whose wavelengths before modulation are in the measurement range of the spectrometer.

If all the $\beta_{m}\left(\omega^{\prime}\right)$ terms in Eq. (9) are substituted with $\beta_{m}\left(\omega_{0}\right)$, where $\omega_{0}$ is the center circular frequency of the chromatic light, that is using the shape of the broadened monochromatic light pulse at circular frequency $\omega_{0}$ to approximate the shapes of all broadened monochromatic pulses centered at different frequencies; Eqs. (11), (12), (17), and (18) become

$$
\begin{aligned}
\Gamma_{\omega_{0}}\left(\omega^{\prime \prime}\right) & =\sum_{m=2}^{\infty} \frac{\beta_{m}\left(\omega_{0}\right)}{m !} \omega^{\prime \prime m} \\
F^{\prime}\left(L, \omega^{\prime \prime}\right) & =F_{0}\left(\omega^{\prime \prime}\right) \exp \left(-j \Gamma_{\omega_{0}}\left(\omega^{\prime \prime}\right) L\right) \\
m^{\prime}\left(L, t-t_{d}\right) & =\int_{-\infty}^{+\infty} F^{\prime}\left(L, \omega^{\prime \prime}\right) \exp \left(j \omega^{\prime \prime}\left(t-t_{d}\right)\right) \mathrm{d} \omega^{\prime \prime} \\
M^{\prime}\left(L, t-t_{d}\right) & =\left|m^{\prime}\left(L, t-t_{d}\right)\right|^{2}
\end{aligned}
$$

respectively, and Eq. (21) thus becomes

$$
s^{\prime}(t)=\int_{-\infty}^{+\infty} P_{n}\left(0, t_{d}\right) M^{\prime}\left(L, t-t_{d}\right) \mathrm{d} t_{d}
$$

which is a convolution of $P_{n}(0, t)$ and $M^{\prime}(L, t)$, i.e.,

$$
s^{\prime}(t)=P_{n}(0, t) * M^{\prime}(L, t) .
$$


Now the system described in Eq. (27) is a linear time invariant (LTI) system and $M^{\prime}(L, t)$ is the impulse response of the system. For a specific dispersive fiber, time $t$ and wavelength $\lambda$ are related by

$$
t=\beta_{1}\left(\frac{2 \pi c}{\lambda}\right) L
$$

The output of the spectrometer in Eqs. (21) and (27) can also be expressed as functions of wavelength,

$$
\begin{aligned}
& s_{\lambda}(\lambda)=\int_{-\infty}^{+\infty} P_{n}\left(0, t_{d}\right) M_{\lambda}\left(L, \lambda, t_{d}\right) \mathrm{d} t_{d}, \\
& s_{\lambda}^{\prime}(\lambda)=P_{n \lambda}(0, \lambda) * M_{\lambda}^{\prime}(L, \lambda),
\end{aligned}
$$

where $M_{\lambda}\left(L, \lambda, t_{d}\right)=M\left(L, t, t_{d}\right), M_{\lambda}^{\prime}(L, \lambda)=M^{\prime}(L, t)$, and $P_{n \lambda}(0, \lambda)=P_{n}(0, t)$.

The output $s_{\lambda}(\lambda)$ in Eq. (29) is the spectral density function of the incoming light acquired by the spectrometer. Thus by experimentally acquiring the output $s(t)$, the spectrum is determined.

\section{Analysis}

\section{A. Accuracy}

In this subsection, the spectrum measurement errors are analyzed through numerical simulation. In the simulation, three fibers with different dispersion properties are used. For all the fibers, the derivatives of the propagation constant $\beta$ with orders higher than 3 are ignored. The value of the group-velocity dispersion (GVD), the second order dispersion $\beta_{3}$, the dispersion parameter $D$ and the dispersion slope $S$ at $1550 \mathrm{~nm}$ are listed in Table 1. $D$ and $S$ are defined by Eqs. (31) and (32),

$$
\begin{aligned}
& D=-\frac{2 \pi c}{\lambda^{2}} \beta_{2}, \\
& S=\frac{\mathrm{d} D}{\mathrm{~d} \lambda},
\end{aligned}
$$

respectively. The first fiber has very large $\beta_{3}$; the second fiber has a dispersion property similar to standard single-mode fiber; the third fiber has constant GVD. The pulses used in the simulations are Gaussian pulses with pulse width of 1 or $0.1 \mathrm{~ns}$, which can

Table 1. Dispersion Properties of the Fibers Used in Simulation

\begin{tabular}{ccccc}
\hline $\begin{array}{c}\text { Fiber } \\
\text { Index } \\
\text { Number }\end{array}$ & $\begin{array}{c}\beta_{2} \\
\left(\mathrm{ps}^{2} / \mathrm{km}\right)\end{array}$ & $\begin{array}{c}\beta_{3} \\
\left(\mathrm{ps}^{3} / \mathrm{km}\right)\end{array}$ & $\begin{array}{c}D \\
\left(\mathrm{ps} \mathrm{nm}^{-1} \mathrm{~km}^{-1}\right)\end{array}$ & $\begin{array}{c}S \\
\left(\mathrm{ps} \mathrm{nm}^{-2} \mathrm{~km}^{-1}\right)\end{array}$ \\
\hline 1 & -595 & 25.5 & 466 & 15.1 \\
2 & -22.5 & 0.128 & 17.6 & 0.0559 \\
3 & -12.8 & 0 & 10.0 & 0.0133 \\
\hline
\end{tabular}

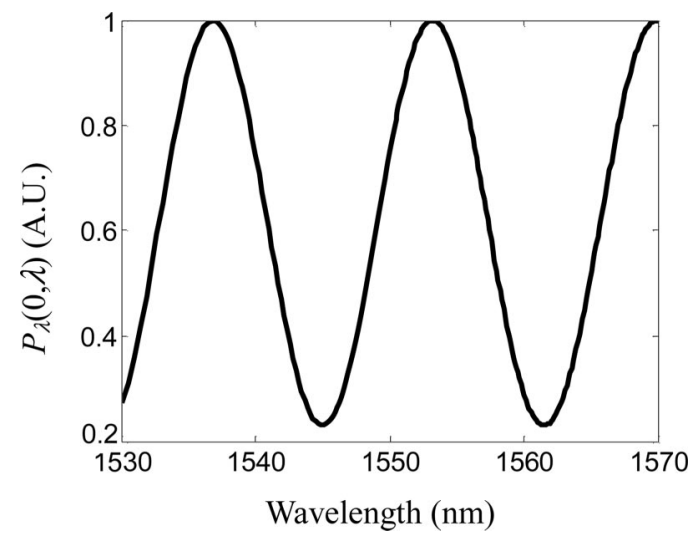

Fig. 2. Calculated spectrum of a FP sensor with a cavity length of $73 \mu \mathrm{m}$.

be realized using commercially available devices. A calculated Fabry-Perot (FP) interferometric sensor spectrum as shown in Fig. 2 is used as the spectrum to be measured in the simulations.

In the following analysis, two types of errors are investigated. One is caused by the operation principle of the spectrometer, which affects the accuracy of the spectrometer. It is defined as the difference between the normalized result from the spectrometer expressed in Eq. (29) and the true spectral density function $P_{\lambda}(0, \lambda)$ :

$$
\Delta_{1}=\left(\int_{-\infty}^{+\infty} P_{n}\left(0, t_{d}\right) M_{\lambda}\left(L, \lambda, t_{d}\right) \mathrm{d} t_{d}\right)_{\text {normalized }}-P_{\lambda}(0, \lambda),
$$

where $P_{\lambda}(0, \lambda)=P(0, \omega)$.

Two reasons cause this error. The first is from the frequency dependent modulation of spectrum $P\left(0, \omega^{\prime}\right)$ by $1 / \beta_{2}\left(\omega^{\prime}\right) L$ expressed in Eq. (20). This can be explained as the nonuniform distribution of the pulses resulting from the fact that $\beta_{1}\left(\omega^{\prime}\right)$ is not a linear function of $\omega^{\prime}$. As discussed in the mathematic model, the output of the spectrometer $s(t)$ or $s_{\lambda}(\lambda)$ could be described as the superposition of all the nonuniformly broadened and delayed monochromatic light pulses whose corresponding wavelengths before the pulse modulation are in the spectrometer's measurement range. The location of each monochromatic light pulse in the time domain is determined by its group delay $\beta_{1}\left(\omega^{\prime}\right) L$. Therefore $s(t)$ or $s_{\lambda}(\lambda)$ has a relatively larger value where the density of the pulses is higher. In Fig. 3 the simulated output $s_{\lambda}(\lambda)$ for an incoming light with a constant spectral density function is presented. The part of error caused by this reason can be partially corrected through amplitude normalization. The correction will have better results where the higher order dispersion parameters are smaller and the width of the broadened monochromatic light pulse is narrower. The second cause of the error is directly related to the broadened pulse width. Each of the broadened pulses has a finite pulse width that 


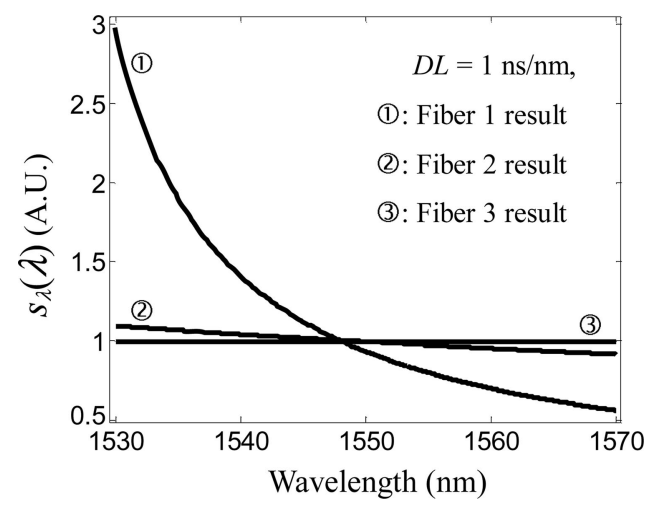

Fig. 3. Spectrometer output $s_{\lambda}(\lambda)$ corresponding to a spectrum with constant spectral density.

causes blur in the output by the superposition of the pulses. This error cannot be corrected by normalization. All these conclusions are confirmed by the numerical simulation results. The error $\Delta_{1}$ in the normalized simulation results using an accurate model [normalized results of Eq. (29)] are presented in Fig. 4. It illustrates that the error $\Delta_{1}$ could not be completely canceled out through normalization. The error is larger when the fiber has larger higher order dispersion. In addition, when the broadened pulse has narrower pulse width in time domain, less error is introduced to the results. When $1 \mathrm{~ns}$ pulse is used [the situation in Figs. 4(a), 4(c), and 4(e)], the corresponding broadened pulse width is $\sim 1 \mathrm{~ns}$; when $0.1 \mathrm{~ns}$ pulse is used [the situation in Figs. 4(b), 4(d), and $4(\mathrm{f})$ ], the corresponding broadened pulse width is $0.11 \mathrm{~ns}$. The errors in the results shown in Figs. 4(b), $4(\mathrm{~d})$, and 4(f) are smaller than the corresponding ones in Figs. 4(a), 4(c), and 4(e).

In addition, to reduce the measurement error, the GVD of the fiber used in the system should not have different signs in the measurement range, i.e., the $\beta_{1}$ of the fiber is monotonously increasing or decreasing in order that lights with different wavelengths are delayed according to the increase or decrease of light frequency or wavelength. Moreover, the modulation pulse should have a spectral width not comparable to the frequency of the light so that the shape of the light pulse will not have severe distortion or destruction after broadening. Only under these condition, $s_{\lambda}(\lambda)$ truly represents the spectrum of the light.

Another error $\Delta_{2}$ is generated during the derivation of the approximate model as presented in Eqs. (23) (27). The error is defined as the difference between the normalized results from Eqs. (29) and (30),

$$
\begin{aligned}
\Delta_{2}= & \left(P_{n \lambda}(0, \lambda) * M_{\lambda}^{\prime}(L, \lambda)\right)_{\text {normalized }} \\
& -\left(\int_{-\infty}^{+\infty} P_{n}\left(0, t_{d}\right) M_{\lambda}\left(L, \lambda, t_{d}\right) \mathrm{d} t_{d}\right)_{\text {normalized }} .
\end{aligned}
$$

The error $\Delta_{2}$ in the normalized results using the approximate model [normalized results of Eq. (30)] are presented in Fig. 5. It indicates that the approximate model will give more accurate results when the broadening of the pulse or the higher-order dispersion is small. Especially when GVD is constant, the approximated model turns into an accurate model and the error $\Delta_{2}$ reduces to 0 . Furthermore, in all the simulation results presented, the errors from the approximation $\Delta_{2}$ are much less than the corresponding errors $\Delta_{1}$. This could be explained as follows. The pulses used in the simulation have a negligible spectral width compared to the light frequency. The pulse broadening is then less determined by those higher order dispersion parameters. So in the measurement range of the spectrometer, the monochromatic light pulses with different wavelengths can be treated as uniformly broadened.

\section{B. Resolution}

The spectral resolution of a spectrometer is usually defined by the minimum wavelength difference between two adjacent peaks with the same intensity in one spectrum, which can be separated by the spectrometer. As shown in Fig. 6, two lights with different wavelengths are located at $\lambda_{1}$ and $\lambda_{2}$, respectively. The spectrum of the light is the superposition of the two pulses. In the following analysis, for simplicity, the pulses are still assumed to be Gaussian type. Then the spectral resolution limit is defined by the wavelength difference of two Gaussian pulses when their $1 / e$ intensity points are overlapped.

The estimation of the spectral resolution can be conducted as follows. The modulation signal $m_{0}(t)$ is a Gaussian pulse with a width of $2 T_{0}$ measured at the $1 / e$ intensity point,

$$
m_{0}(t)=\exp \left(-\frac{t^{2}}{2 T_{0}^{2}}\right)
$$

The origin of the time axis is located at the center of the pulse. Ignoring the derivatives of the propagation constant $\beta$ with orders higher than 2, from Eq. (18) the shape of the broadened pulse is

$$
M^{\prime}(L, t)=\frac{1}{1+\left(\frac{\beta_{2}\left(\omega_{0}\right) L}{T_{0}^{2}}\right)^{2}} \exp \left(-\frac{t^{2}}{T_{0}^{2}\left(1+\left(\frac{\beta_{2}\left(\omega_{0}\right) L}{T_{0}^{2}}\right)^{2}\right)}\right) .
$$

In Eq. (36), the origin of the time axis is also located at the center of the pulse. The half width of the broadened pulse is expressed as

$$
T_{0}^{\prime}=T_{0} \sqrt{1+\left(\frac{\beta_{2}\left(\omega_{0}\right) L}{T_{0}^{2}}\right)^{2}} .
$$

According to the spectral resolution criterion mentioned above, the spectral resolution of the new spectrometer can be estimated by

$$
r_{\lambda}=\frac{2 T_{0}^{\prime}}{D_{L}\left(\lambda_{0}\right)}=2 \sqrt{\left(\frac{T_{0}}{D_{L}\left(\lambda_{0}\right)}\right)^{2}+\left(\frac{\lambda_{0}^{2}}{2 \pi c T_{0}}\right)^{2}}
$$




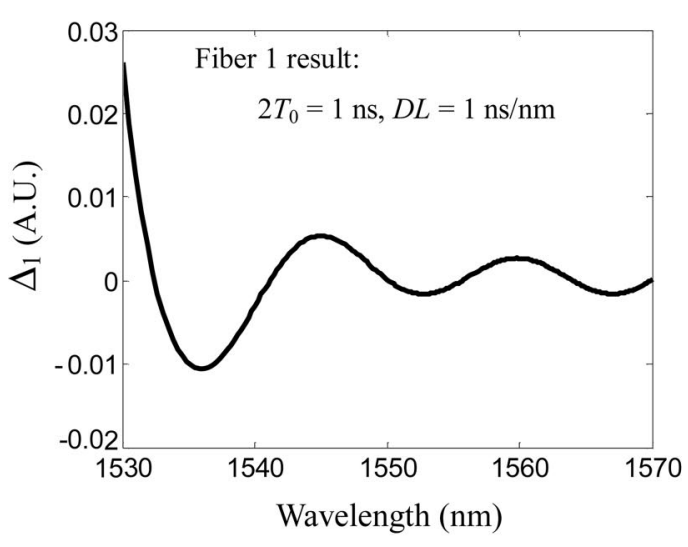

(a)

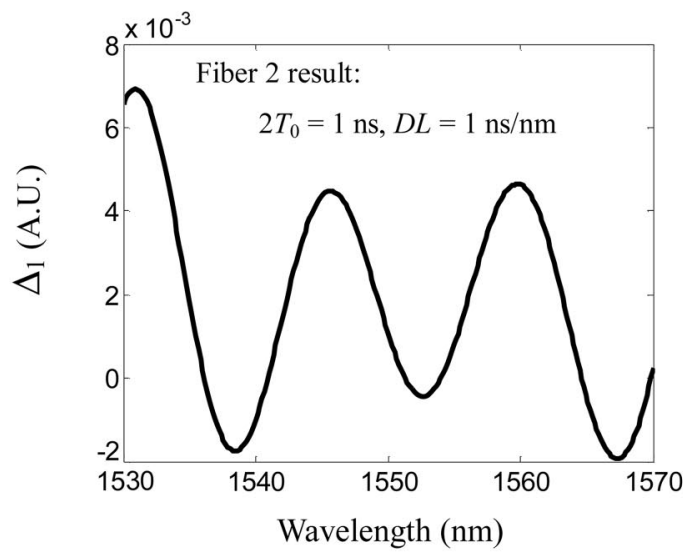

(c)

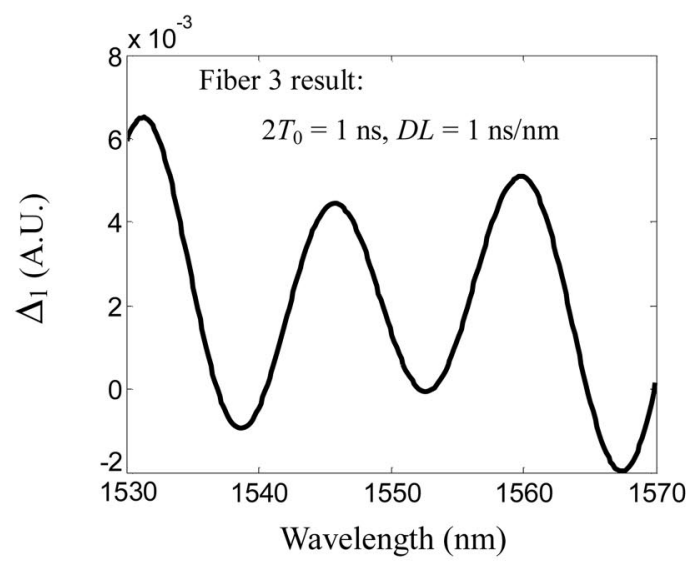

(e)

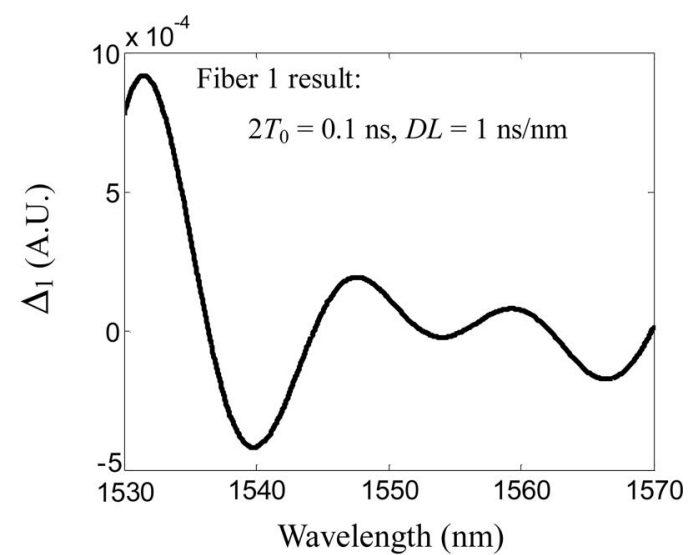

(b)

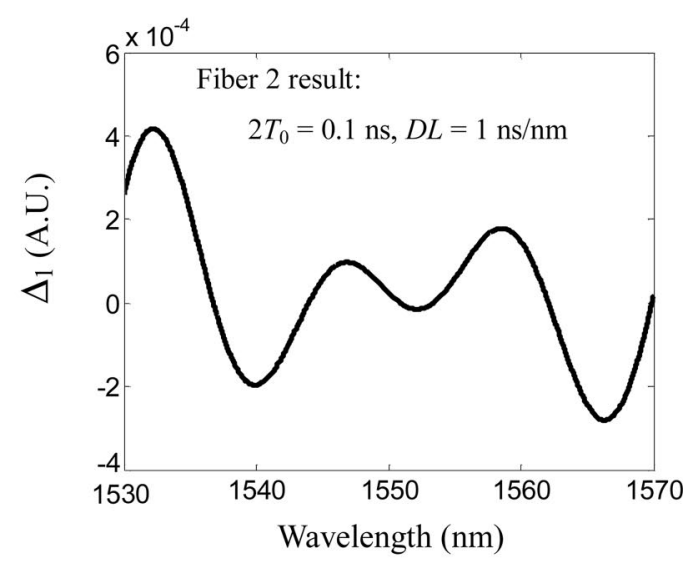

(d)

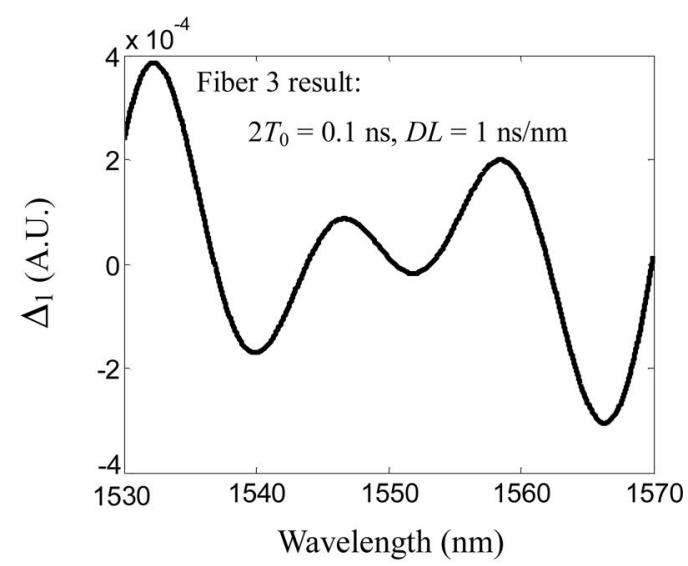

(f)

Fig. 4. Simulation results of the error $\Delta_{1}$.

where

$$
\begin{aligned}
D\left(\lambda_{0}\right) & =-\frac{2 \pi c}{\lambda_{0}^{2}} \beta_{2}\left(\omega_{0}\right), \\
D_{L}\left(\lambda_{0}\right) & =D\left(\lambda_{0}\right) L
\end{aligned}
$$

are the dispersion parameter of the fiber at wavelength $\lambda_{0}$ and the total amount of dispersion intro- duced by the dispersive fiber, respectively. In the above equations, $c$ is the speed of light in vacuum. The spectral resolution $r_{\lambda}$ in Eq. (38) has its minimum:

$$
r_{\lambda m}=2 \sqrt{\frac{\lambda_{0}^{2}}{\pi c\left|D_{L}\left(\lambda_{0}\right)\right|}},
$$




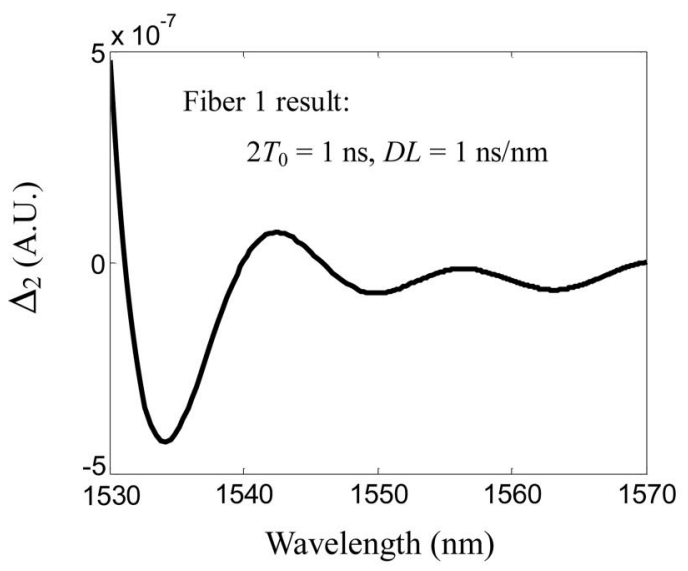

(a)

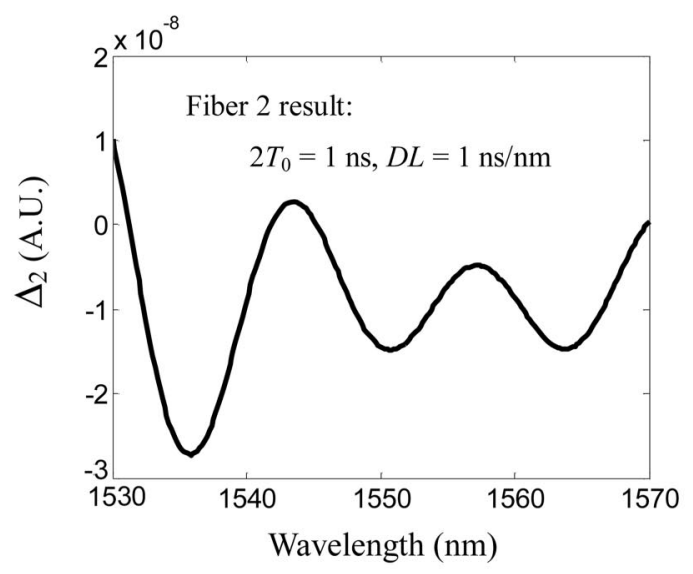

(c)

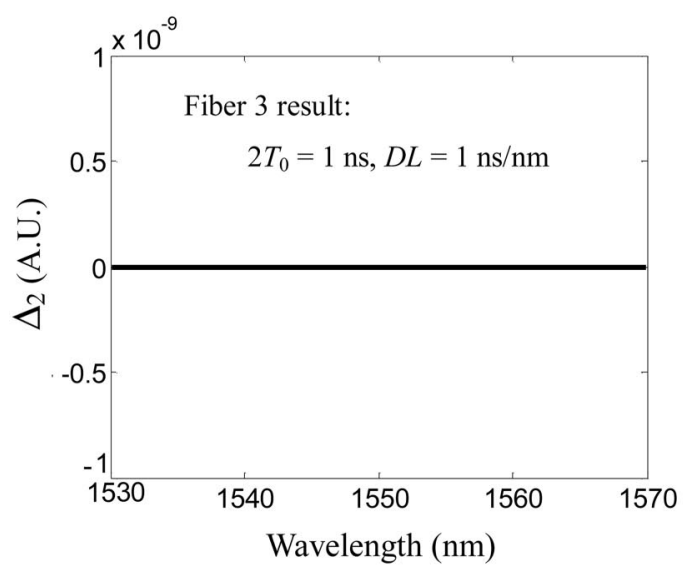

(e)

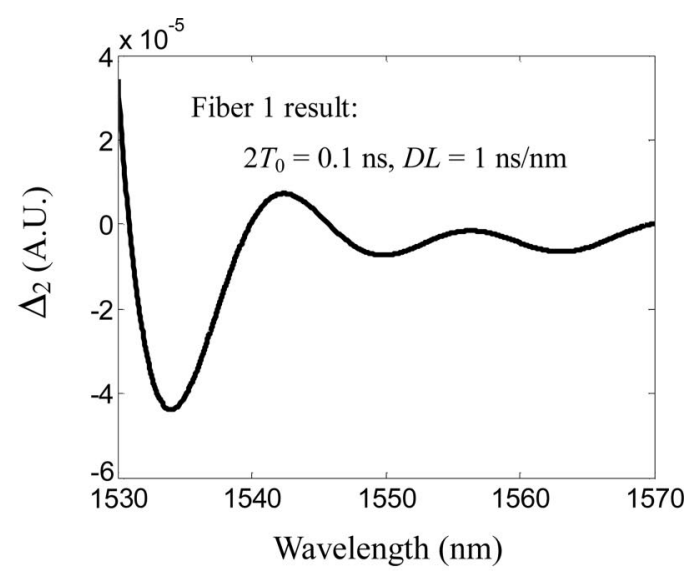

(b)

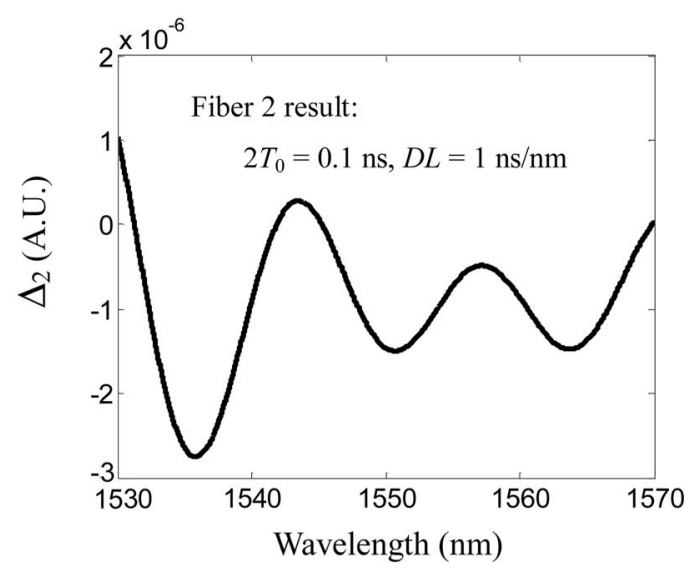

(d)

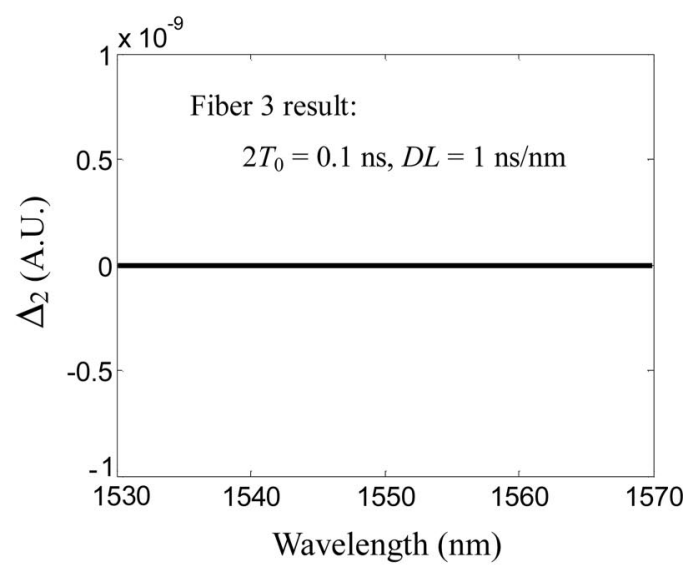

(f)

Fig. 5. Simulation results of the error $\Delta_{2}$.

$$
T_{0}=\sqrt{\frac{\lambda_{0}^{2}}{2 \pi c}\left|D_{L}\left(\lambda_{0}\right)\right|} .
$$

The above analysis indicates that for specific wavelength $\lambda_{0}$ the spectral resolution is determined by the total amount of dispersion $D_{L}\left(\lambda_{0}\right)$ of the dispersive fiber and the initial pulse width $2 T_{0}$ of the light pulse. When the amount of dispersion is fixed, there exists an optimal pulse width at which the best spectrum measurement resolution is achieved as shown by Fig. 7(a). This is so because the output pulse broadening caused by the inherent bandwidth of the pulse profile and the GVD of the dispersion element is dominant when the monochromic input optical pulse is sufficiently narrow. Figure 7(b) shows the relationship between the spectral resolution and the total dispersion of the fiber for different pulse widths. It is indicated that, for each fixed initial pulse width $2 T_{0}$, the 


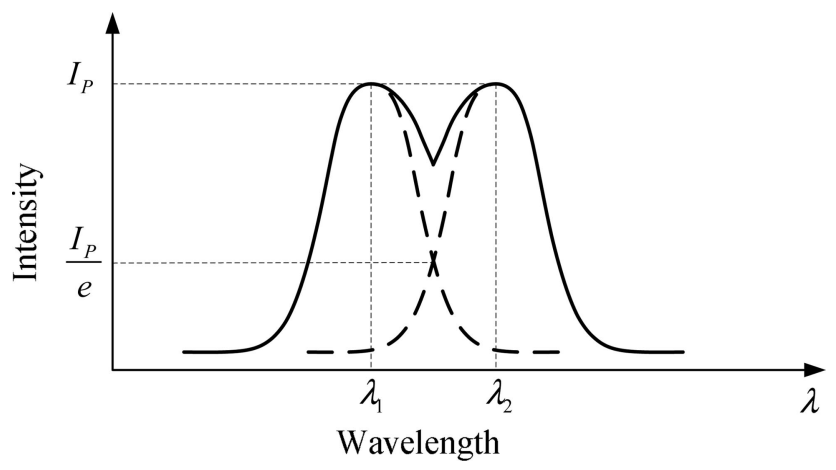

Fig. 6. Definition of spectral resolution.

spectral resolution decreases rapidly when the dispersion is smaller than the value determined by Eq. (42). The relation between the best spectral resolution and the corresponding initial pulse width $2 T_{0}$ is illustrated in Fig. 8(a). In Fig. 8(b), the best spectral resolution is presented as a function of the dispersion. If the dispersion in a spectrometer is $2 \mathrm{~ns} / \mathrm{nm}$, for example, the best resolution is $0.07 \mathrm{~nm}$ when $0.1 \mathrm{~ns}$ Gaussian pulse is used.

In our experiment [3], the amount of dispersion is $\sim 500 \mathrm{~ns} / \mathrm{nm}$ and the pulse width is $\sim 6 \mathrm{~ns}$ (measured at half intensity position). The pulse shape is close to

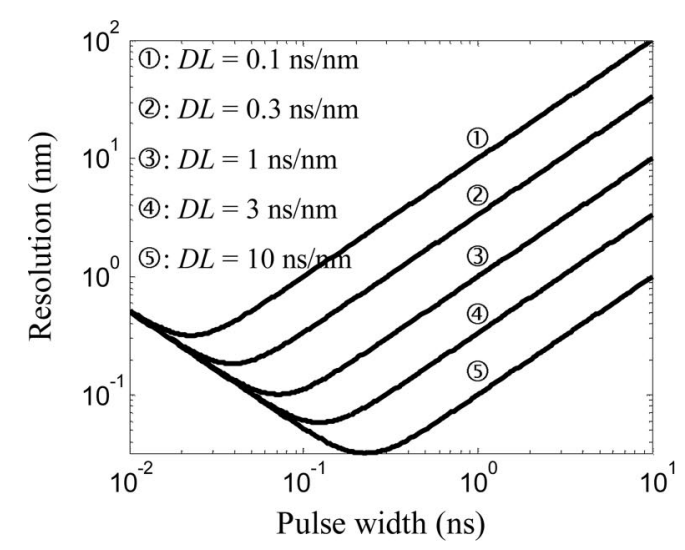

(a)

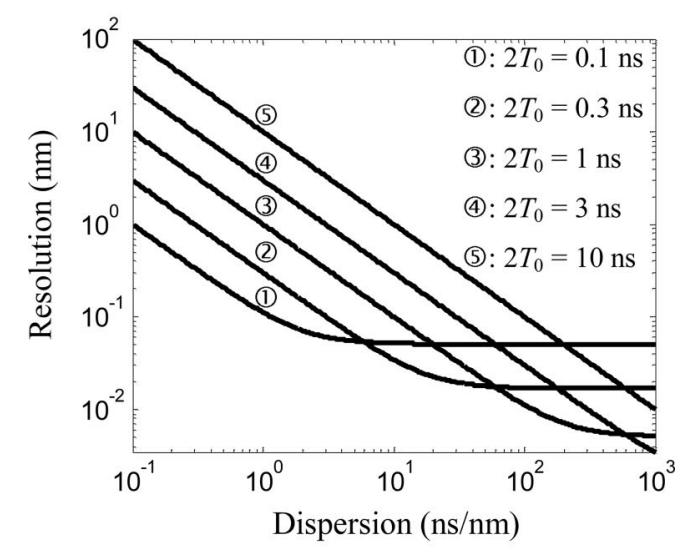

(b)

Fig. 7. Resolution analysis at $1550 \mathrm{~nm}$ using Gaussian pulse.

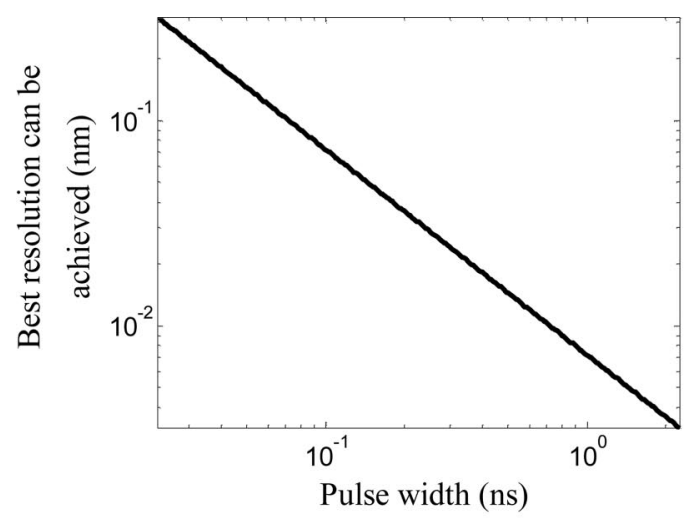

(a)

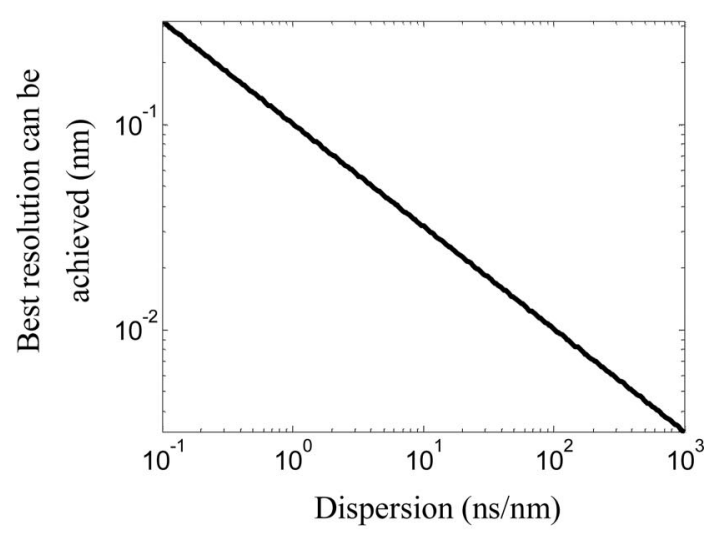

(b)

Fig. 8. Best spectral resolution at $1550 \mathrm{~nm}$ using Gaussian pulse.

the rectangle window function, which is determined by the pulse generator. The experimental spectral resolution then becomes $15 \mathrm{~nm}$ at $1550 \mathrm{~nm}$, whereas the spectral resolution from the model is $13.4 \mathrm{~nm}$. This difference mainly results from the distortion in the pulse shape introduced by the erbium-doped fiber amplifier (EDFA) which is used for loss compensation in the optical path.

\section{Speed}

The speed of the spectrometer is defined as the number of spectrum frames acquired per unit time. It is mainly determined by the width of the broadened chromatic light pulses in the time domain. The time interval between two consecutive pulses must be larger than this pulse width; otherwise measurement error could be introduced by the overlap between two consecutive pulses. Three parameters affect the width of the broadened output pulses. They are the width of the spectrum to be measured, the amount of dispersion introduced by the dispersive fiber, and the properties of the modulation pulse. For a Gaussian pulse, the intensity at $t= \pm 3 T_{0}$ decreases to $10^{-4}$ of the peak intensity. The width of the chromatic light pulse measured at the $10^{-4}$ intensity point can be roughly estimated by 


$$
T_{s}=\Delta \lambda D_{L}\left(\lambda_{0}\right)+6 T_{0}^{\prime},
$$

where $T_{s}$ is the output pulse width and $\Delta \lambda$ is the $-3 \mathrm{~dB}$ spectral width of the incoming light. The maximum speed is defined as the speed at which the $10^{-4}$ intensity points of the two adjacent output pulses are overlapped. Therefore the maximum speed is estimated by

$f_{m}=\frac{1}{T_{s}}=\left(\left(\Delta \lambda+6 \sqrt{\left(\frac{T_{0}}{D_{L}\left(\lambda_{0}\right)}\right)^{2}+\left(\frac{\lambda_{0}^{2}}{2 \pi c T_{0}}\right)^{2}}\right) D_{L}\left(\lambda_{0}\right)\right)^{-1}$.

When the half initial pulse $T_{0}$ equals the value presented in Eq. (42), the best spectral resolution is reached for a given dispersion value. Figure 9(a) illustrates the maximum speed as a function of the spectral width. In Fig. 9(b), the relationship between the maximum speed and the best resolution is presented.

Figure 9(a) indicates that the maximum speed decreases as the spectral width increases or as the

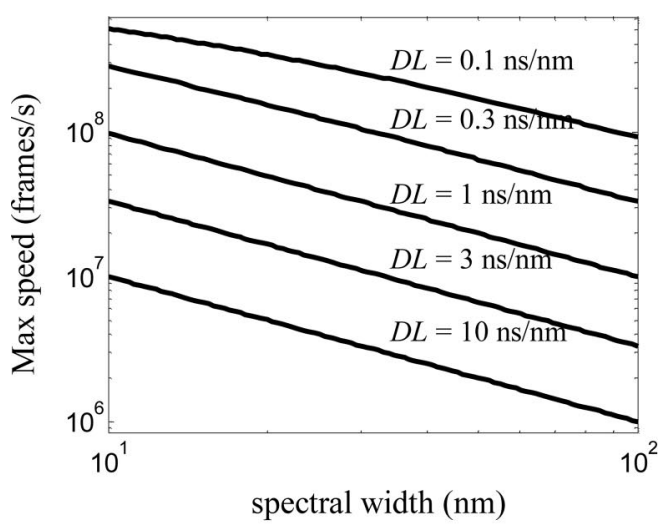

(a)

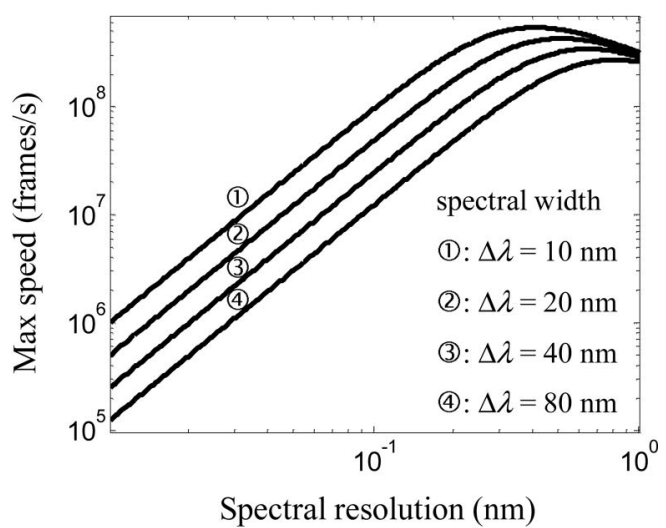

(b)

Fig. 9. Relationships between the maximum spectrum acquisition rate, the spectral width, and the spectral resolution when the best spectral resolution for each dispersion value is reached. total amount of dispersion increases. For all five dispersion values, the measurement speeds are above $10^{6}$ frames/s when the spectral widths are less than $100 \mathrm{~nm}$. Figure 9(b) shows that there is a trade-off between the best achievable spectral resolution and the maximum measurement speed. In addition, for each spectral width, a highest value of the maximum measurement speed exists. On the left side of each curve to its highest value, the increase of the dispersion that results in the spectral resolution improvement lowers the maximum measurement speed; on the right side of each curve to its highest value, the pulse broadening causes the decrease of the maximum measurement speed.

\section{Conclusion}

In this paper a mathematical model for the HSFOS is developed. This model accurately describes the relationship between the optical spectrum of the incoming light and the dispersed time-domain signal. The output of the spectrometer can be treated as the superposition of all the frequency-dependent broadened and delayed monochromatic light pulses whose wavelengths before pulse modulation are in the measurement range of the spectrometer. In order that the output has a shape that can represent the spectrum of the incoming light, two requirements should be fulfilled. One is that the GVD of the fiber used in the spectrometer should not have different signs in the measurement range, i.e., the $\beta_{1}$ of the fiber is monotonously increasing or decreasing. The other is that the modulation pulse should have a spectral width not comparable to the frequency of the light so that the shape of the light pulse will not have severe distortion or destruction after broadening. Further, the effects of the key parameters such as the fiber dispersion and the pulse width on the performance of the spectrometer, e.g., the measurement accuracy, the spectral resolution, and the spectrum acquisition speed, are investigated. To reduce the measurement error, the width of the broadened pulse should be decreased and the results should be normalized. In addition, the fiber with small higher-order dispersion should be chosen. The trade-off between the speed and the spectral resolution makes it necessary to carefully determine the two parameters according to the requirements of an application.

\section{References}

1. B. Qi, G. Pickrell, J. Xu, P. Zhang, Y. Duan, W. Peng, Z. Huang, W. Huo, H. Xiao, R. G. May, and A. Wang, "Novel data processing techniques for dispersive white light interferometer," Opt. Eng. 42, 3165-3171 (2003).

2. B. Yu, A. Wang, G. Pickrell, and J. Xu, "Tunable-optical-filterbased white-light interferometry for sensing," Opt. Lett. 30, 1452-1454 (2005).

3. F. Shen and A. Wang, "Frequency-estimation-based signalprocessing algorithm for white-light optical fiber Fabry-Perot interferometers," Appl. Opt. 44, 5206-5214 (2005).

4. M. Han, Y. Zhang, F. Shen, G. Pickrell, and A. Wang, "Signal- 
processing algorithm for white-light optical fiber extrinsic Fabry-Perot interferometric sensors," Opt. Lett. 29, 1736-1738 (2004).

5. Y. Wang, M. Han, and A. Wang, "High-speed fiber-optic spectrometer for signal demodulation of interferometric fiber-optic sensors," Opt. Lett. 31, 2408-2410 (2006).
6. D. Marcuse, "Pulse distortion in single-mode fibers," Appl. Opt. 19, 1653-1660 (1980).

7. D. Marcuse, "Propagation of pulse fluctuations in single-mode fibers," Appl. Opt. 19, 1856-1861 (1980).

8. D. Marcuse, "Pulse distortion in single-mode fibers. Part 2," Appl. Opt. 20, 2969-2974 (1981). 